\title{
Patterns for Session-Based Access Control
}

\author{
Eduardo B. Fernandez ${ }^{1)}$ and Günther Pernul ${ }^{2)}$ \\ Dept. of Computer Science and Engineerging \\ Florida Atlantic University \\ Boca Raton, FL 33431, USA \\ ed@cse.fau.edu \\ ${ }^{2)}$ Department of Information Systems \\ Universität Regensburg \\ Universitätsstraße 31, Regensburg, Germany \\ guenther.pernul@wiwi.uni-regensburg.de
}

\begin{abstract}
The concept of session, the context under which a user accesses resources is very important to apply access control. We present first the Access Session pattern for describing how sessions can limit the rights of a user. We then combine this pattern with two existing access control patterns. First we consider a pattern for Session-Based Role-Based Access Control, intended for organizations in which job functions form the basis for privilege assignments. Then, we present a Session-Based Attribute-Based Access Control pattern for organizations in which accesses are controlled based on values of user attributes and object properties. Since the general properties of those patterns have been described earlier we emphasize the additional effect of using sessions.
\end{abstract}

\section{Introduction}

It is important to develop systems where security has been considered at all stages of design, which not only satisfy their functional specifications but also satisfy security requirements. To do this we need to start with high-level models that represent the security policies of the institution. There are three models currently used by most systems: the access matrix, the Role-Based Access Control (RBAC) model, and the multilevel model.

One of the first security models was the access matrix. The basic access matrix [Lam71] included the tuple $\{\mathrm{s}, \mathrm{o}, \mathrm{t}\}$, where $\mathrm{s}$ indicates a subject or active entity, $\mathrm{o}$ is the protected object or resource, and $\mathrm{t}$ indicates the type of access permitted. [Har76] proved security properties of this model using the socalled HRU (Harrison-Ruzzo-Ullman) model. In that model users are allowed to delegate their rights (discretionary property, delegatable authorization), implying a tuple $\{\mathrm{s}, \mathrm{o}, \mathrm{t}, \mathrm{f}\}$, where $\mathrm{f}$ is a Boolean copy flag indicating if the right is allowed to be delegated or not. A predicate was added to the basic rule to allow content-based authorization [Fer75], becoming $\{\mathrm{s}, \mathrm{o}, \mathrm{t}, \mathrm{p}, \mathrm{f}\}$, where $\mathrm{p}$ is the predicate (the predicate could also include environment variables). Patterns for the basic rule and for the tuple $\{\mathrm{s}, \mathrm{o,t}, \mathrm{p}, \mathrm{f}\}$ were given in [Fer01a, Sch06]. The rule could also include the concept of Authorizer (a), becoming \{a,s,o,t,p,f\} [Fer81] (Explicitly Granted Authorization). RBAC [San96] can be considered a special interpretation of the basic authorization model, where subjects are roles instead of individual users. We presented two varieties of RBAC patterns in [Fer01a] and [Sch06]. Subsequently, several variations and extensions of these models have appeared. We presented a variation called MetadataBased Access Control, which later we renamed Attribute-Based Access Control (ABAC) [Pri04, Pri05]. 
ABAC can be seen in two ways:

- A specialization of the model $\{\mathrm{s}, \mathrm{o}, \mathrm{t}, \mathrm{p}\}$, where $\mathrm{p}$ is a predicate which depends on attribute values.

- $\quad$ A variant where s and o are defined by descriptors which depend on attribute values.

In this paper we present a general pattern for Access Session as a building block and two patterns for RBAC and ABAC making use of the Access Session pattern, the Session-Based RBAC and the Session-Based ABAC pattern. Sessions describe an environment where the rights available to a user can be dynamically limited based on the context in which the user is acting. The pattern diagram of Figure 1 shows the relationships between these patterns. For example, adding a condition to Basic Authorization results in Content-Based Authorization, using the concept of session results in sessionbased models, and so on. Note that RBAC is in general not delegatable. All these patterns define authorization rules and they need a reference monitor for their enforcement; we don't show it in this diagram for simplicity (see [Sch06] for the corresponding pattern). The double-lined patterns are the ones presented here. We assume the reader to know basic security concepts and these patterns are intended for system designers trying to add security to their designs.

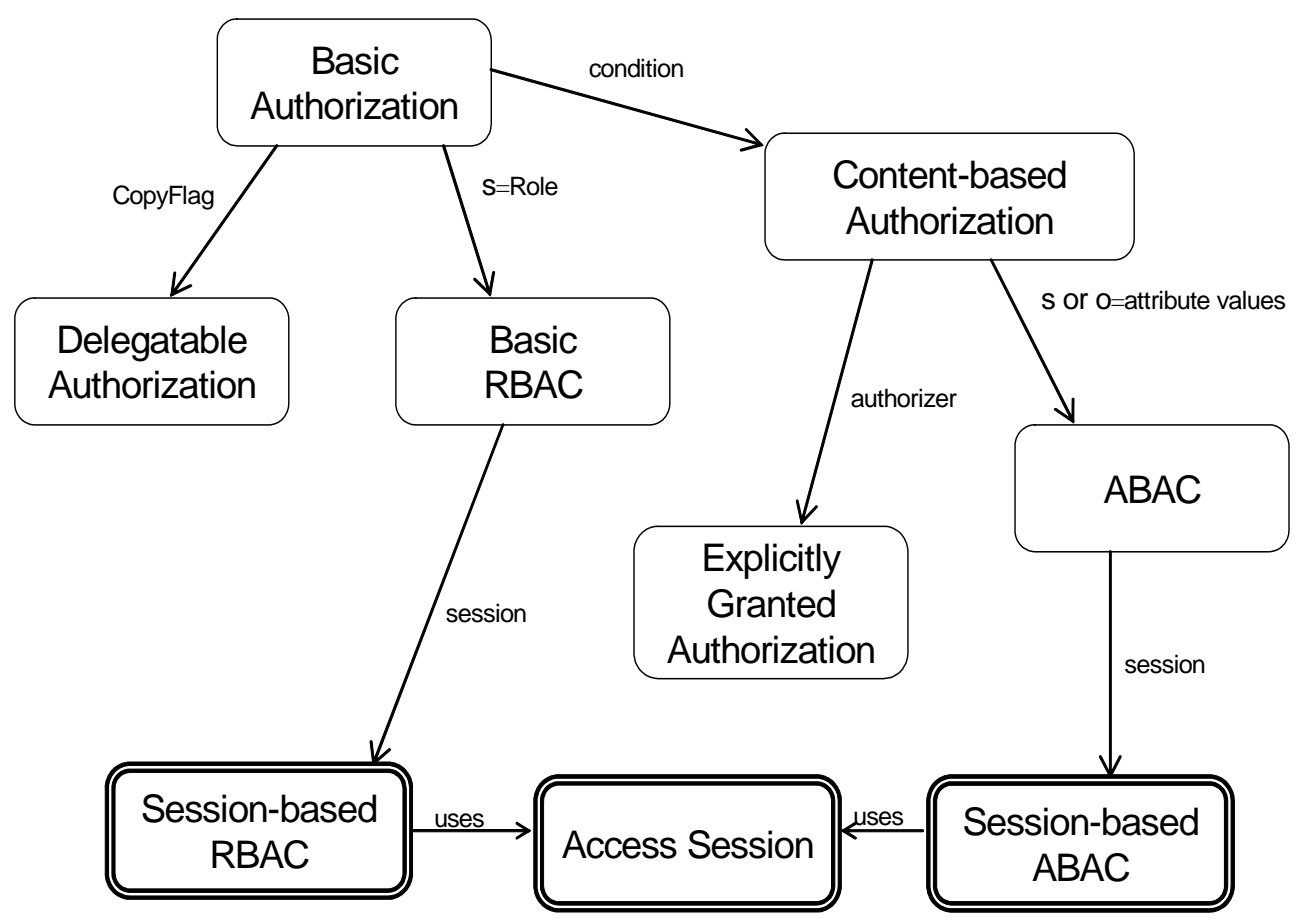

Figure 1. Relationships between access control patterns 


\section{Access Session}

Provide a context in which a security subject can access resources. Depending on the context he is using, the rights of a user may vary.

\subsection{Example}

Lisa is a secretary in a medical organization but sometimes she helps in the laboratory to perform patient tests. As a secretary she has access to patients' information such as name, address, SSN, etc. This is necessary so she can bill them and their insurance companies. In the lab she has access to anonymized patient test results. Combining the accesses provided by her two jobs in one window she can associate test results to names, which violates patient privacy.

\subsection{Context}

Any environment where we need to control access to computing resources and where users can be classified according to their jobs, groups, departments, assignments, or tasks.

\subsection{Problem}

A given user may be authorized to access a system because she needs to perform several functional activities. However, for a particular access only those privileges should be active which are necessary to perform the intended task. This is an application of the principle of least-privilege and necessary to prevent the user from misusing the system (intentionally, accidentally by performing an error, or without knowledge and tricked to do so, for example through a Trojan Horse attack). Additionally this would potentially restrict damage in case of session hijacking. A successfully attacking process would not have all privileges of a user available but only the active subset.

The following forces will affect the solution:

- Subjects may have many rights directly or indirectly through the execution contexts that they need for their tasks. Using all of them at one time may result in conflicts of interest and security violations. We need to restrict the use of those rights depending on the application or task the subject is performing.

- In the context of an interaction we can make the access to some functions implicit, thus facilitating the use of the system and preventing errors that may result in vulnerabilities. For example, some editors or other tools could be implicitly available in some sessions.

- It is not convenient to make subjects reauthenticate every time they request a new resource. Once the subject is authenticated, this condition should remain valid during the whole session.

\subsection{Solution}

Define a unit of interaction, a session, which has a limited lifetime, e.g. between login and logoff of a user or between the beginning and the end of a transaction. When a user logs on and after authentication, the session activates some execution contexts with only a subset of the authorizations she possesses. It should be the minimal subset which is needed for the user or transaction to perform the intended task. Only those rights are available within the session. A subject can be in several sessions at the same time; however, in every session only the necessary rights are active.

\section{Structure}


Figure 2 shows the class model of the Access Session pattern. Classes Subject and Session have the obvious meaning. The class ExecutionContext contains the set of active rights that the user may use within the session.

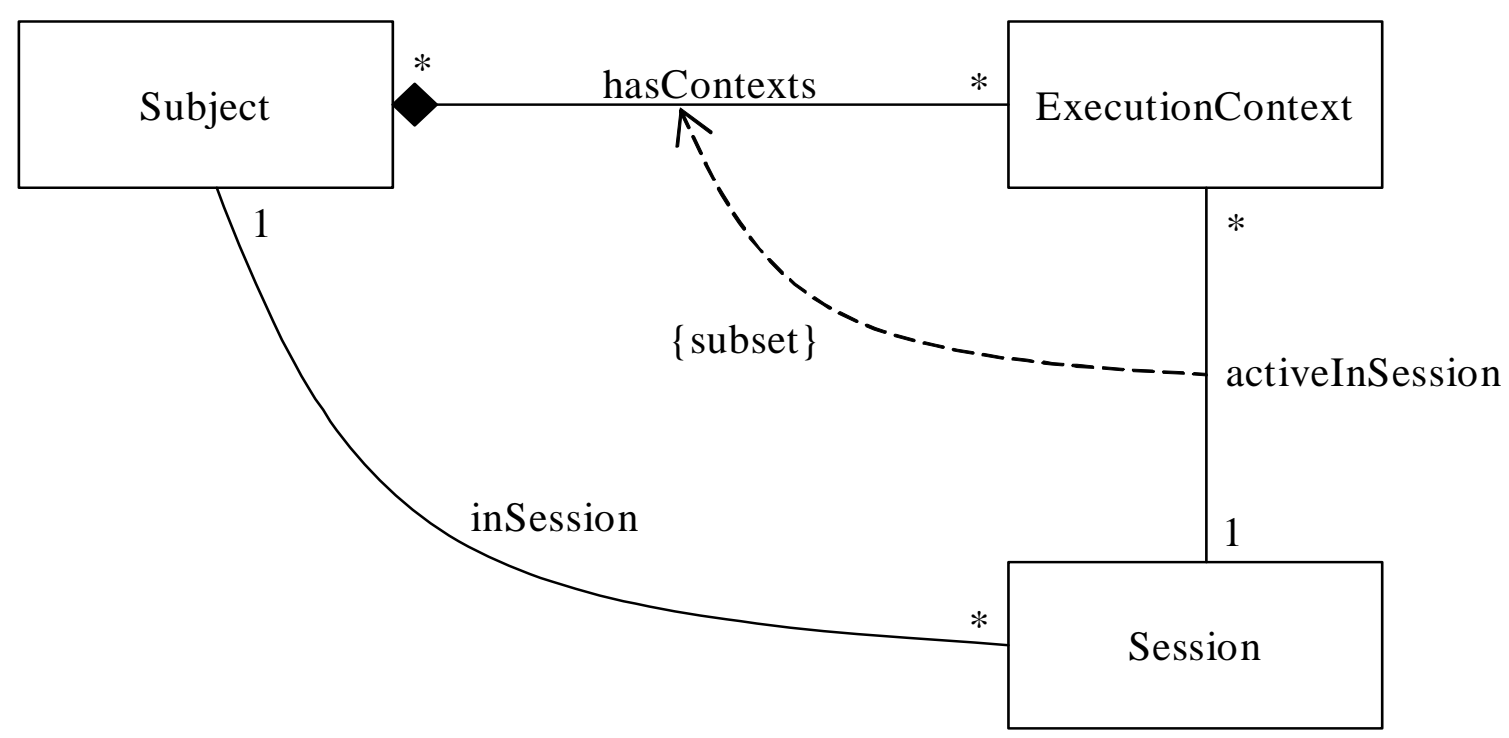

Figure 2: Class model for Access Session pattern

\section{Dynamics}

Figure 3 shows the use case Open (Activate) a session. A subject logs on using some of his contexts and the logon interface authenticates the subject. The box indicates some authentication dialog or protocol. After the subject is authenticated, the interface creates a session object and returns a handle to the subject.

\subsection{Implementation}

Based on institution and application policies define which contexts (implying specific rights) should be used in each task and grant them to the corresponding subject. The rights should be selected using the least privilege principle and there should be no contexts with excessive rights, e.g. the administrator rights should be divided into smaller sets.

\subsection{Example resolved}

Lisa can log on a secretary or as a lab assistant but she cannot combine these activities in one session. Now she cannot relate results to patient names.

\subsection{Known uses}


- Session Access is part of the RBAC standard proposal by NIST which later has been adopted by the American National Standards Institute, International Committee for Information Technology Standards (ANSI/INCITS) as ANSI INCITS 359-2004 [Fer01b].

- Multics [Sum97] used execution contexts (based on projects) to limit access rights. Session Access is implemented in the security module CSAP [Dri03] of the Webocrat System in conjunction with an RBAC policy.

- Views in relational databases can be used to define sets of rights. Controlling the use of views by users can control their use of rights in sessions. This is done for example in Oracle and DB2, where SQL can be used to define restricted views [Elm03].

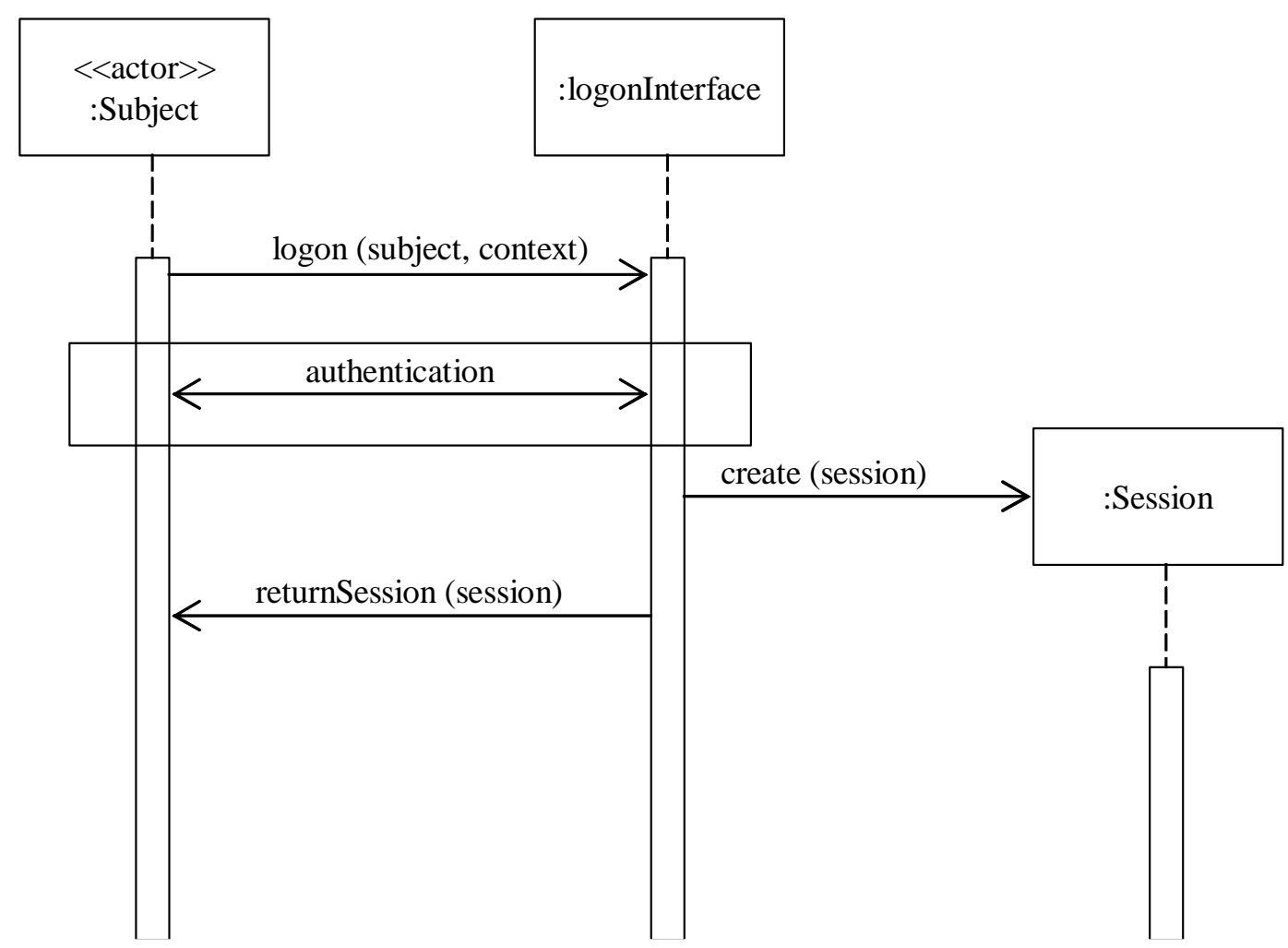

Figure 3. Sequence diagram for use case 'Open a session'

\subsection{Consequences}

This pattern has the following advantages:

- We can give to each context only the needed rights according to its function and we can invoke in a session only those contexts that are needed for a given task.

- We can exclude combinations of contexts that might result in possible access violations or conflicts of interest.

- Any functions can be made implicit in a session.

- Once a subject starts a session it doesn't have to be reauthenticated. Its status is kept by the session.

A possible disadvantage: 
If we need to apply fine-grained access it might be inefficient to include many contexts to perform complex activities.

\subsection{Related patterns}

The Access Session pattern is used in the Session-Based RBAC and ABAC patterns, discussed later.

The Session pattern of [Yod97] created a session object that defined a namespace to hold all the variables that need to be referenced by many objects. P. Sommerlad remade this pattern as a Security Session [Sch06], intended to prevent a user to be reauthenticated every time he accesses a new object. A pattern with a similar objective is Abstract Session [Pry00]: When an object's services are invoked by clients, the server object may have to maintain state for each client. The server creates a session object that encapsulates state information for the client. The server returns a pointer to the session object. However, none of these patterns considers limitation of rights. Our pattern is an extension of those patterns emphasizing the effect of a session as a limiter of rights.

\section{Session-Based Role-Based Access Control}

Allow access to resources based on the role of the subject and limit the rights that can be applied at a given time based on the contexts (roles) defined by the access session.

\subsection{Example}

John is a developer in a project. He is also a project leader in another project. As a project leader he can evaluate the performance of the members of his project. He combines his two roles and adds several flattering evaluations about himself in the project where he is a developer. Later his manager thinking that they came from the project leader of that project, gives John a big bonus.

\subsection{Context}

Any environment where we need to control access to computing resources, where users can be classified according to their jobs or their tasks, and where we assign rights to the roles needed to perform those tasks..

\subsection{Problem}

In an organization a user may play several roles. However, for each access the user must act only within the authorizations of a single role (i.e. within the context of the role) or combinations of roles that do not violate institution policies. How to restrict subjects to follow the policies of the institution?

In addition to the forces defined for the Access Session pattern, the following forces apply to the solution:

- People in institutions have different needs for access to information, according to their functions. They may have several roles associated with specific functions or tasks.

- We want to help the institution to define precise access rights for its members so that the least privilege policy can be applied when they perform specific tasks..

- Users may have more than one role and we may want to enforce policies such as separation of duty, where a user cannot be in two or more specific roles in the same session. 


\subsection{Solution}

A subject may have several roles. Each role collects the rights that a user can activate at a given moment (execution context), while a session controls the way of using roles and can enforce role exclusion at execution time.

\section{Structure}

The structure of the session-based RBAC is shown in the class diagram given in Figure 4. The class Role is an intermediary between subject and object holding all authorizations a user possesses while playing the role and acts here as an execution context. Within a Session, only a subset of the roles assigned to a Subject may be activated, i.e. only those necessary to perform the intended task. Roles may be composed according to a Composite pattern [Gam94], where higher-level roles inherit rights from the lower-level roles.

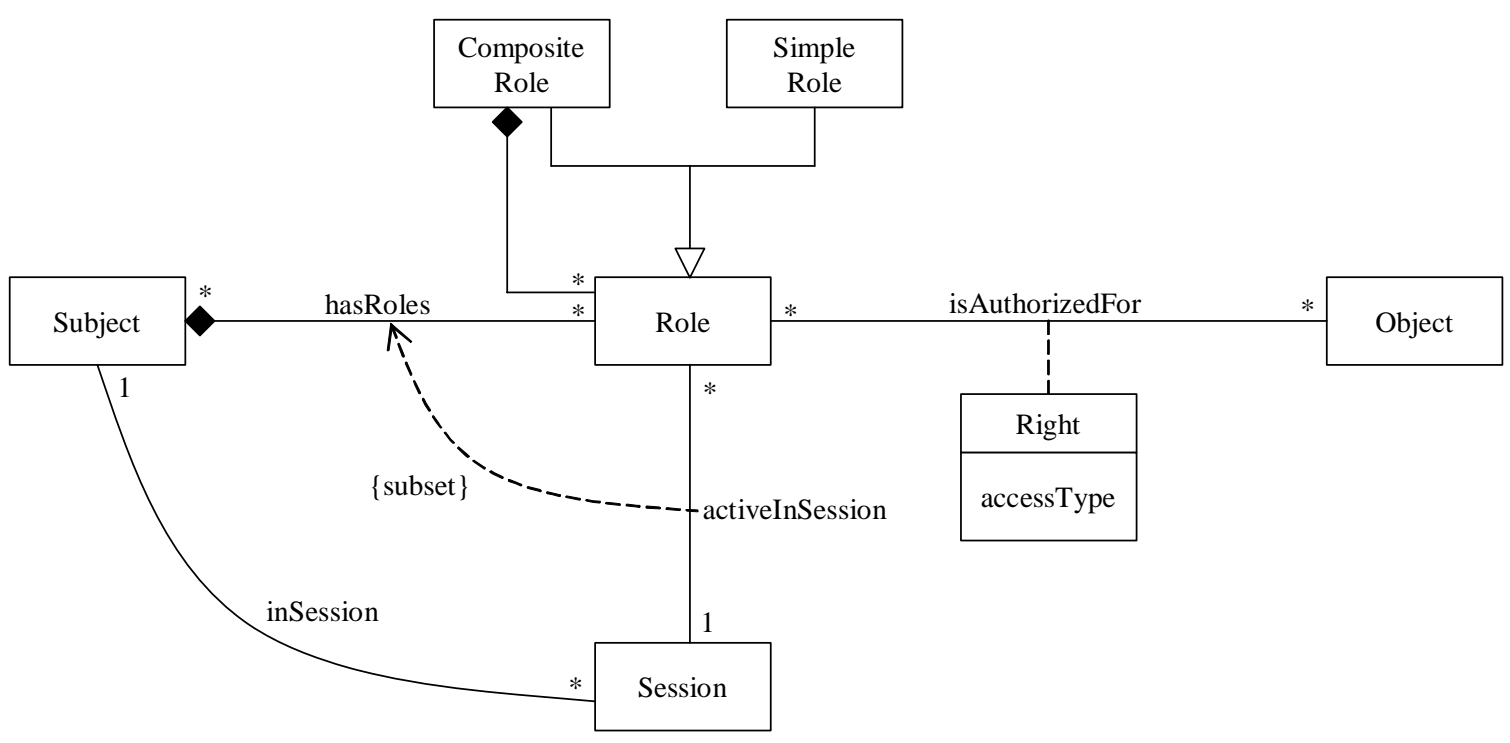

Figure 4. Class model for the Session-Based RBAC

\subsection{Implementation}

See Section 5 for an example of a real implementation.

- Determine the roles the system should contain (role catalog), according to the user functions or tasks.

- Collect lists of incompatible roles and use these lists when a session is started (static constraints). These constraints can be defined using OCL or some other formal language. 
- Determine the number of roles which may be active within a session (dynamic constraints).

- When a user opens a session she must declare what roles she intends to use and the system will open the corresponding session or refuse to do so in case of conflicts.

\subsection{Example resolved}

When John logs on the project where he is a developer he only gets the rights for a developer and cannot add evaluations. When he logs on in the project where he is a project leader he can only evaluate the members of his group. Now he only gets legitimate evaluations.

\subsection{Known uses}

The structure and dynamics of the session-based RBAC as given above are implemented in the security module CSAP [Dri03] of the Webocrat system. Webocrat is a portal supporting EDemocracy which was developed within the European Webocracy project (FP5-IST-1999-20364) between 2000-2003.

Views in relational databases can be used to define sets of rights. Controlling the use of views by roles can control the use of rights in sessions. This is done for example in Oracle and DB2, where SQL can be used to define restricted views [Elm03].

\subsection{Consequences}

In addition to the advantages mentioned for the Access Session pattern, among the advantages of this pattern we have:

- Users can activate more than one session at a time for functional flexibility (some tasks may require multiple roles).

- Fine-grained rights can be assigned to roles to enforce a need-to-know policy.

- We can exclude roles that violate institution policies when a session is open.

Possible disadvantages include:

- Additional conceptual complexity to define which roles can be used together and which should be mutually exclusive.

\subsection{Related patterns}

This pattern is a combination of the Session pattern described earlier and the RBAC pattern [Sch06]. As indicated earlier, structuring of roles can be represented by a Composite pattern.

\section{Session-Based Attribute-Based Authorization}

Allow access to resources based on the attributes of the subjects and the properties of the objects but limit the rights that can be applied at a given time based on the context defined by the access session.

\subsection{Example}

Meili is a teenager who likes movies and subscribes to several movie services through the Internet. She logs in a central portal where she can reach sets of movies. Sometimes she gets movies that she finds offensive or inappropriate (pornographic, racist, plain stupid). She doesn't have much time to read details about the movies in advance and some of them don't even have good descriptions so 
reading about the movies is not a good approach. She would like some kind of filter according to her characteristics and her preferences. Also the portal may be breaking the law in making available to her some of these movies.

\subsection{Context}

Dynamic systems supporting a large set of objects and subjects in which the structure of the subjects changes rapidly, such as web-based information systems, e-government and e-business portals. In this environment there is the need to control access to computing resources and the subjects may not be preregistered. We want to give access to resources based on characteristics of the subjects such as groups to which they belong, company for which they work, biological characteristics such as age, etc.

\subsection{Problem}

As indicated access may depend on the age or other attributes of a user. In this case, privilege assignments to the user cannot be done statically by a security administrator but automatically by the system based on the value of some of the attributes, e.g. "DateOfBirth" . As the user gets older or changes functions his authorization state changes automatically. Access rights might even depend on an external attribute, such as "physical location" of a user in a mobile environment. In this case the authorization state changes automatically when the user moves around. At the object's side, metadata such as the scope of a document, or the MPAA rating of a movie are examples of properties. All these constraints can be applied through predicates in the rules [Fer81], but it is difficult to have a variety of prepackaged rules for the typical cases.

The solution is constrained by the following forces:

- We need to limit the rights of subjects that are in a variety of groups or roles, or have special characteristics. Unrestricted access might allow policy or law violations.

- This control should not imply an extra burden for the security administrator.

- This control should not imply a significant performance overhead.

- The environment is very dynamic and changes should be easy to make.

\subsection{Solution}

Access rights are based on the comparison of values of selected attributes of subjects and properties of objects (so called subject and object descriptors). In this pattern descriptors are a construct to somehow "group” objects and subjects dynamically, not explicitly by an administrator but implicitly by their attribute or property values. This grouping may result in unpredictable sets of rights that may violate security policies. A session delimits the rights that can be applied at a given moment; that is, the subject attributes define a context for access rights. .

\section{Structure}

Figure 5 shows the class diagram for the solution. A Subject Descriptor is formed by applying Qualifiers to Attribute Values. A Session selects some specific values as execution context that defines the Subject rights.

\subsection{Implementation}

See Section 5 for an example of a real implementation.

1) Select an appropriate package to convey the subject's credentials including attributes. Examples would be attribute certificates [Mor06, Opp00] or Kerberos tickets. 
2) Select an implementation to express the object's attributes. Candidates could be standards on metadata resource discovery, such as the Dublin Core Metadata Initiative [DCM].

3) Define an enforcement mechanism for the rights defined in contexts. See for example [Cor04].

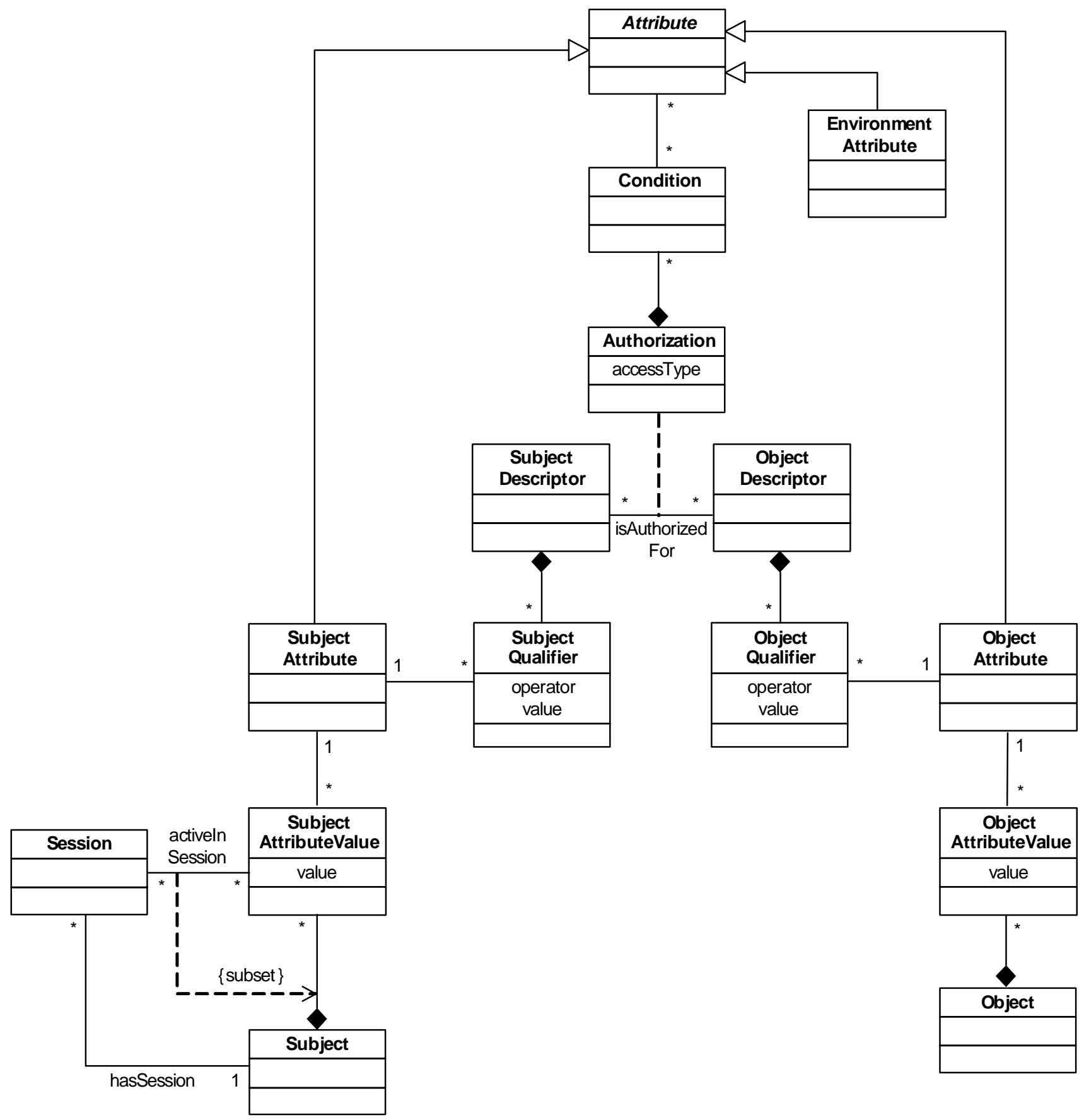

Figure 5. Class model for the Session-Based ABAC pattern 


\subsection{Example resolved}

The portal implemented an ABAC model. Now when Meili opens a session she is given access to contexts with sets of preselected movies according to her preferences and restricted according to legal aspects and to the services she has paid for.

\subsection{Known Uses}

Session-based ABAC is implemented as an alternative to RBAC in the security module CSAP [Dri03] of the Webocrat system. A similar pattern is also used in the authorization system of the .NET component framework [LaM02] and in AAIs (authentication and authorization infrastructures), such as Permis [Cha03] and Shibboleth [Shi].

The XML standard XACML [Del05, OAS03] uses attributes of subjects and objects for the specification of access control policies. As shown in the $\mathrm{UCON}_{\mathrm{ABC}}$ [Par04], ABAC may also have potential for digital rights management.

\subsection{Consequences}

The advantages of this pattern include:

- The rights of subjects that belong to a variety of groups, roles, or have special attributes can be limited by restricting them to use specific contexts selected by sessions.

- This control does not imply an extra burden for the security administrator because the contexts can be defined by application designers according to application policies.

- This control does not imply a significant performance overhead because changing from one context to another just means changing a set of rights.

- Changes in access restrictions can be easily accommodated by defining new contexts or deleting existing contexts.

Possible disadvantages are:

- Higher complexity. Although the contexts are defined by others, it is hard for administrators to know who has access to what.

- There might still be some performance overhead if we need to switch often between contexts.

\subsection{Related patterns}

Figure 1 shows the relationship of this pattern to other access control patterns. As indicated credentials such as certificates are frequently used to request access [Mor06].

\section{Using session-based access control as a service}

In this section we show by means of two sequence diagrams how the patterns described above can be embedded into a general authentication, authorization and access control service. Such a service can be called by any application or process having the need to authenticate the users and to provide session-based access control. In the following it is assumed that the service provides both sessionbased RBAC and session-based ABAC and the client application requesting the service must chose between the two. 
Figure 6 shows a sequence diagram for the interaction of a requesting client process and the sessionbased access control service. In order to hide the complexity of the subsystems, in the sequence diagram we use the Facade pattern [Gam94] as a uniform interface for calling applications.

In order to be able to access a resource, a valid session object must be requested by the calling application (or user process). This starts with some sort of initialization process during which the client application first requests from the authentication facade of the security service an authentication service. In the example shown in figure 6 a password service is returned but also other services may be available. Second is the request for an authorization service. In the example RBAC is returned, and the initialization phase is finished. Next is the actual user authentication, role selection and the session establishment. During user authentication the client application provides to the password service <user-id, pwd>. The password service interacts with a userDM and in case of successful log-in a user object is created and a reference to the object (aUser) is returned to the calling client application.

A valid session can only be established in the case the user application activates at least one role from the set of possible roles for the user. This starts by calling the method getAssignedRoles of the user object. In case of a valid userID all available roles for a particular user are determined and returned by the role data module (RoleDM) and for each role a transient role object is created by the RBAC service. Next from the set of possible roles the user selects a subset and the RBAC service calls the corresponding method to activate the roles.

At this stage the user object is authenticated and has a set of active roles assigned. These are the only prerequisites for establishing a session. After receiving the request the session service creates a valid session object for which the session-id is returned as a reference for the calling client process. Under a valid session-id the client may act under the context of the session by using the privileges of the selected roles. 


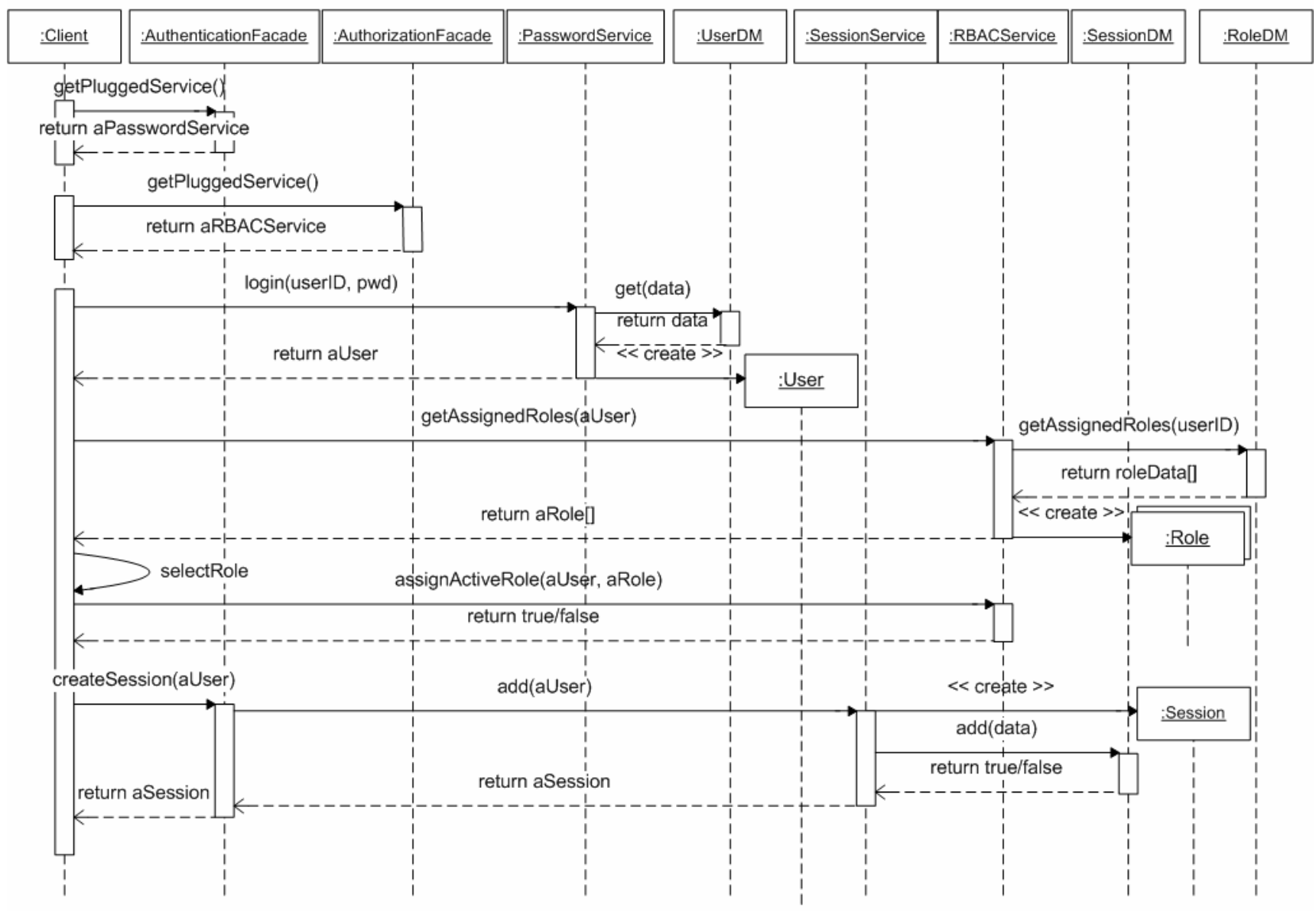

Figure 6. Session establishment

Figure 7 shows an attempt of a client process to access a resource within a valid session. The process starts with calling the method checkAccess with parameters session-id, object-id, operation, i.e. a request of a user wishing to access a certain object by using a predefined operation and this all within the context of an established session. First, the validity of the session is checked, then the session object is used by the RBACService in order to get the user's active roles within this session. Next, the user's permissions are determined by retrieving all the permissions assigned to the active roles. Finally, the RBACService checks whether there is a permission for the tuple <object, operation>. In the case there is one, the access will be granted, otherwise denied. 


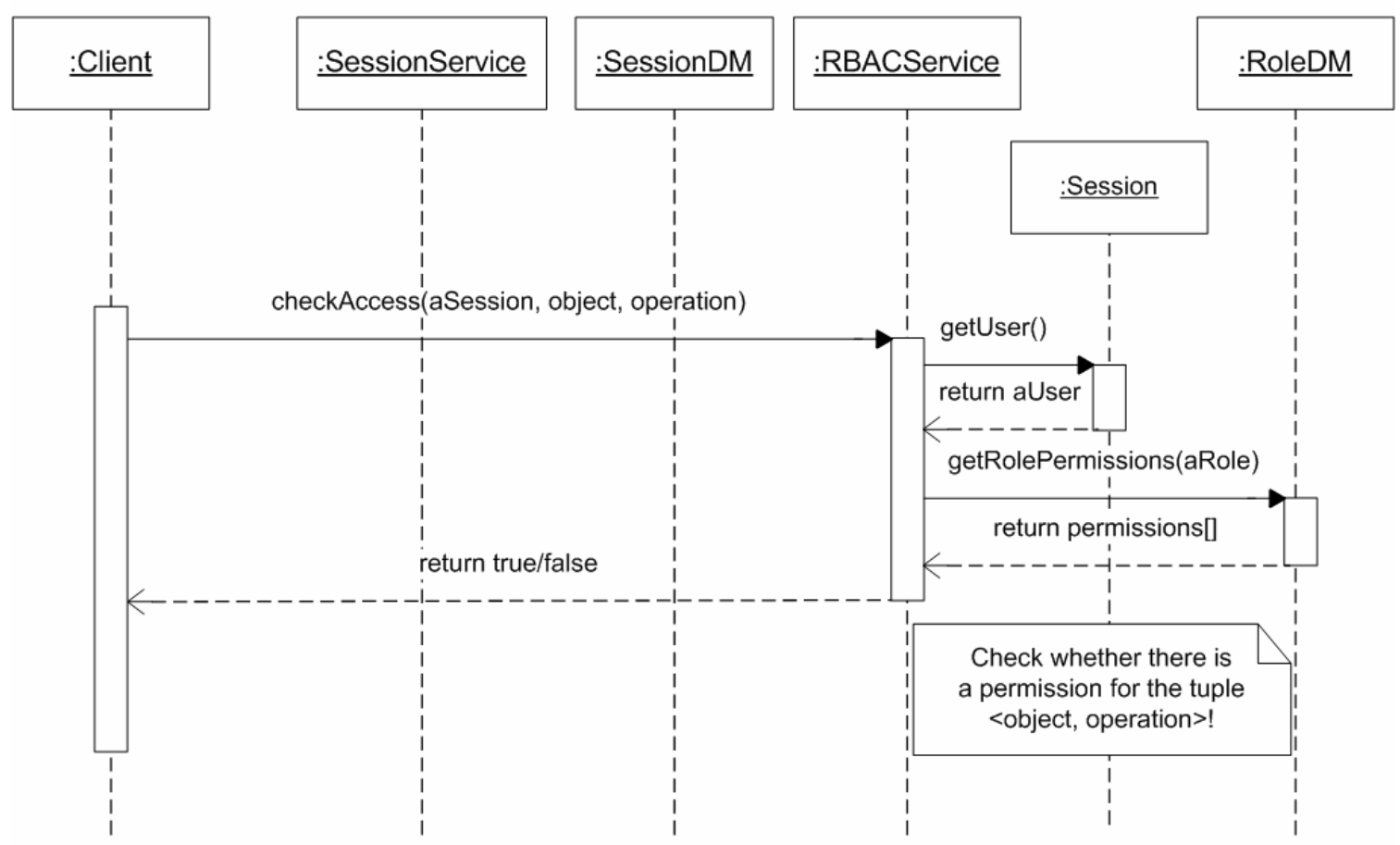

Figure 7. Permission approval

\section{Conclusions}

We have shown patterns to describe the effect of sessions on access control models. We presented first the Access Session, which describes the basic concept of session as a limiter of rights. We then combined this pattern with the patterns of two access control models to show its effect on them. Finally we showed an example of a system using the last two patterns as a way to illustrate a real implementation.

\section{Acknowledgements}

We thank our shepherd Michael Weiss for his valuable comments that contributed to improve this paper. The FAU Secure Systems Research Group provided useful improvements and corrections. The work of E. Fernandez was supported by a Federal Earmark grant from DISA, administered by Pragmatics, Inc. The work of G. Pernul was partly supported by the European Commission DG INFSO under the IST program, Webocracy, contract No. IST-20364. 


\section{References}

[Cha03] D.W.Chadwick and A. Otenko, "The PERMIS X.509 role based privilege management infrastructure”, Future Generation Computer Systems, vol. 19, No 2, 2003, 277-289.

[Cor04] A. Corradi, R. Montanari, and D. Tibaldi, "Context-based access control management in ubiquitous environments", Procs. Third IEEE Int. Symp. On Network Computing and Applics. (NCA'04.

[DCM] The Dublin Core Metadata Initiative. http://www.dublincore.org.

[Del05] N. Delessy, E. B.Fernandez, and T. Sorgente, "Patterns for the eXtensible Access Control Markup Language", Procs. of the Pattern Languages of Programs Conference (PLoP 2005), Allerton Park, IL, September 2005.

[Dri03] F.Dridi, M.Fischer, and G.Pernul, "CSAP -- an adaptable security module for the egovernment system Webocrat". Proc. of the 18th IFIP International Information Security Conference (SEC 2003), Athens, Greece, 26-28 May 2003.

[Elm03] R. Elmasri and S. Navathe, Fundamentals of database systems ( $4^{\text {th }}$ Ed.), Addison-Wesley 2003.

[Fer75] E. B. Fernandez, R. C. Summers, and C. B. Coleman, "An authorization model for a shared data base," Proc. of the 1975 SIGMOD International Conference, ACM, New York, 23-31, 1975.

[Fer81] E. B. Fernandez, R. C. Summers, C. Wood, Database Security and Integrity, AddisonWesley, Reading, Massachusetts, Systems Programming Series, February 1981.

[Fer01a] E. B. Fernandez, and R. Pan, “A pattern language for security models”, Procs. of PLoP 2001.

[Fer01b] D. Ferraiolo, R. Sandhu, S. Gavrila, D.R.Kuhn, and R. Chandramouli, "Proposed NIST standard for Role-Based Access Control”, ACM Trans. on Information and System Security, Vol. 4, No 3, August 2001, 224-274.

[Gam94] E. Gamma, R. Helm, R. Johnson, J. Vlissides. Design Patterns: Elements of Reusable Object-Oriented Software, Addison-Wesley, Boston, Mass., 1994.

[Har76] M.Harrison, W. Ruzzo, J. Ullman, "Protection in Operating Systems", Comm. of the ACM, Vol. 19, No 8, August 1976.

[Lam71] B. W. Lampson, "Protection", Proceedings of the 5th Princeton Conference on Information Sciences and Systems, Princeton, 1971 
[LaM02] B.A.LaMacchia, S. Lange, M. Lyons, R. Martin, and K.T.Price, NET framework security, Addison-Wesley, 2002.

[Mor06] P. Morrison and E.B. Fernandez, “The Credentials Pattern”, accepted for the Pattern Languages of Programs Conference (PLoP 2006).

[OAS03] eXtensible Access Control Markup Language (XACML), Version 1.1. OASIS Community Specification, August 2003. http://www.oasis-open.org/committees/xacml/

[Opp00] R.Oppliger, G. Pernul, and C. Strauss, “ Using Attribute Certificates to implement RoleBased Authorization and Access Control", Proc. of the 4th Conference on "Sicherheit in Informationssystemen" (SIS 2000), Zürich (Switzerland), October 5 - 6, 2000, vdf Hochschulverlag.

[Par04] J.Park, J., and R.Sandhu,” The UCONABC usage control model”, ACM Transactions on Information Systems Security, 7(1), pp. 128-174, February 2004.

[Pri04] T. Priebe, E.B.Fernandez, J.I.Mehlau, and G. Pernul, "A pattern system for access control", in Research Directions in Data and Applications Security XVIII, C. Farkas and P. Samarati (Eds.), Procs of the 18th. Annual IFIP WG 11.3 Working Conference on Data and Applications Security, Sitges, Spain, July 25-28, 2004.

[Pri05] T. Priebe, W. Dobmeier, B. Muschall, and G. Pernul, "ABAC - Ein Referenzmodell für attributbasierte Zugriffskontrolle“, Proc. Sicherheit 2005, 2. Jahrestagung des Fachbereichs Sicherheit der Gesellschaft für Informatik, Regensburg, April 2005.

[Pry00] N. Pryce, “Abstract session: An object structural pattern”, Chapter 7 in Pattern Languages of Program Design 4 (N. Harrison, B. Foote, and H. Rohnert, Eds.). Also in Procs. of PLoP'97.

[San96] R. Sandhu, E.J.Coyne, H.L.Feinstein, and C.E.Youman., "Role-based access control models", Computer , vol. 29 , No2, February 1996, 38-47.

[Sch06] M. Schumacher, E.B. Fernandez, D. Hybertson, F. Buschmann, and P. Sommerlad, Security Patterns: Integrating security and systems engineering, J. Wiley \& Sons, 2006.

[Shi] Shibboleth Project, http://shibboleth.internet2.edu

[Sum97] R. C. Summers, Secure Computing: Threats and Safeguards, McGraw-Hill, 1997

[Yod97] J. Yoder and J. Barcalow, “Architectural Patterns for Enabling Application Security,” Proc. of the $4^{\text {th }}$ Conference of Pattern Languages of Programs (PLoP'97).Also, Chapter 15 in Pattern Languages of Program Design, vol. 4 (N. Harrison, B. Foote, and H. Rohnert, Eds.), Addison- Wesley, 2000. 